\title{
Frequency response of a liquid sloshing in a rotating, laterally oscillating, cylindrical vessel
}

\author{
Eisaku Yokose $^{1, *}$, Yusuke Saito ${ }^{1, * *}$, and Tatsuo Sawada ${ }^{1, * *}$ \\ ${ }^{1}$ Department of Mechanical Engineering, Keio University \\ 3-14-1 Hiyoshi, Kohoku-ku, Yokohama 223-8522, Japan
}

\begin{abstract}
We have investigated the free-surface fluctuations of liquid in a rotating, laterally oscillating, cylindrical vessel. In the experiments, we changed independently both the lateral forcing frequency $f$ and the rotation frequency $\Omega$ of the cylinder. We measured the time-dependent fluid-dynamic pressure instead of the free surface fluctuations. From an FFT analysis of the dynamic pressure, we obtained two significant frequencies: $f-\Omega$ and $f+\Omega$. They are associated, respectively, with waves that rotate in the same direction, and in the opposite direction, relative to the rotation of the vessel. We also examined these results using linearized wave theory.
\end{abstract}

\section{Introduction}

Sloshing is the largely wave-like motion of the free surface of a liquid caused by the oscillation of a vessel. Even if the oscillation is small, the changes in the liquid level may increase. Swirling is the rotation of the free surface of a liquid that takes place when sloshing occurs in an axisymmetric vessel. There are two types of swirling: stable rotations and unstable rotations. Both sloshing and swirling are concerns for oil tanks, membrane-type LNG tankers, liquid-fuel tanks in rockets and missiles, etc. Hutton [1] investigated the motion of fluid in a tank undergoing transverse oscillations, and he predicted the range of frequencies over which swirling occurs. The behavior of the free surface of the liquid in the rotating container was investigated by Rocca et al. [2] and Bauer et al. [3]. Takahara et al. [4] examined the frequency response of nonlinear sloshing in a pitching excited annular cylindrical tank. Numerous theoretical, experimental, and numerical studies of these phenomena have been conducted, as summarized by Ibrahim et al. [5-6].

Ohaba et al. [7] experimentally investigated the frequency response of the lateral sloshing and rotation of the $(1,1)$ mode wave in a cylindrical vessel undergoing uniform rotation around the vertical axis. They found that the direction of the swirling rotation depends on the excitation conditions and the rotation frequency. They pointed out that the swirling generated in a cylindrical vessel undergoing lateral oscillations can be stabilized by the energy dissipation caused by the rotational motion. Saito and Sawada [8] considered the qualitative influence of the rotation frequency of the cylindrical vessel on water sloshing, and they speculated that the rotational motion may stabilize the sloshing fluctuations of the free surface.

\footnotetext{
*e-mail: eskyks-9705@a3.keio.jp

**e-mail: trombone.yusuke@gmail.com

***e-mail: sawada@mech.keio.jp
} 
In the present study, we clarify the dynamic characteristics of swirling in a rotating, laterally oscillating, cylindrical vessel. We measured the time-dependent dynamic pressure of the liquid on the wall of the vessel instead of the free-surface displacement of the liquid. The experimental parameters are the lateral forcing frequency, the rotation frequency of the cylindrical vessel, the liquid depth, and the viscosity of the liquid. We have also carried out a theoretical analysis in order to understand the experimental results.

\section{Experimental apparatus}

The experimental apparatus is shown in Fig. 1. The cylindrical vessel, made of acrylic, has a height of $200 \mathrm{~mm}$ and an inner diameter of $99 \mathrm{~mm}$. It is connected to a DC motor fixed directly to the oscillating table. The cylindrical vessel is caused to oscillate laterally by an oscillation generator. The forcing frequency $f$ of the oscillation and the rotation frequency $\Omega$ produced by the DC motor are both continuously variable. We fixed the amplitude of the lateral oscillation to be $a=1.5 \mathrm{~mm}$ and the liquid depth to be $h=30 \mathrm{~mm}$ throughout the experiments. We used a mixture of water and glycerin as the test liquid so that the viscosity of the liquid can be changed by varying the amount of glycerin relative to water.

The pressure sensor, which is connected to a radio-signal processing system, is embedded in the inner wall of the cylindrical vessel $13 \mathrm{~mm}$ from the bottom. We measured the fluid-dynamic pressure instead of the free-surface displacement because both change in synchrony. Specifically, we recorded the difference $\Delta p$ between the maximum and minimum of the pressure fluctuations measured by the pressure sensor. We used $\Delta p$ as a measure of the magnitude of sloshing and swirling during the lateral oscillations. In addition, we have investigated the frequency components of the free-surface behavior using a Fast Fourier Transform (FFT) analysis.

\section{Linearized theory}

The analytical model we used is shown in Fig. 2. Here, $R$ is the radius of the cylindrical vessel, $\xi$ is the angular velocity of the vessel, $\omega$ is the angular frequency of lateral oscillation,

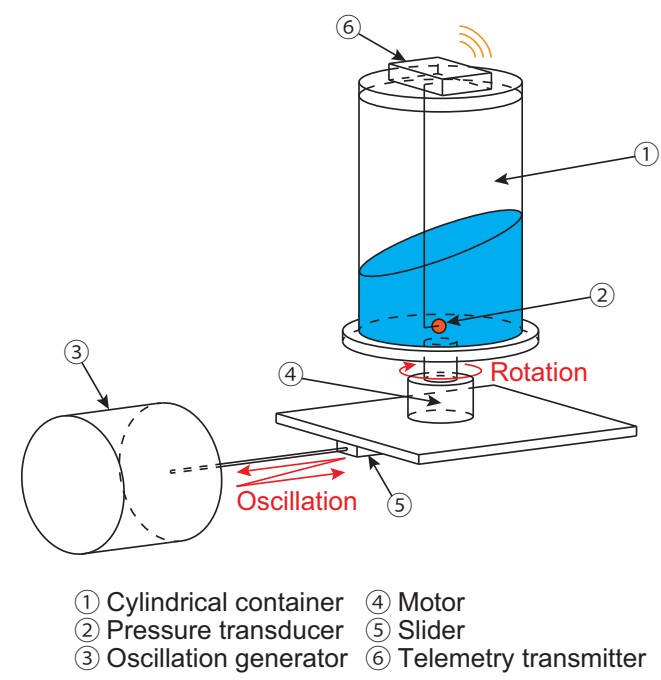

Figure 1. Experimental apparatus 


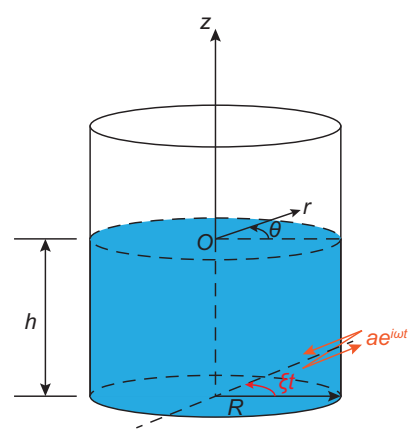

Figure 2. Analytical model of the cylindrical vessel

and $i$ is the imaginary unit, If the motions are irrotational and the fluid is incompressible, a velocity potential $\phi$ exists that satisfies the continuity equation. It also satisfies the Laplace equation in cylindrical coordinates:

$$
\frac{1}{r} \frac{\partial}{\partial r}\left(r \frac{\partial \phi}{\partial r}\right)+\frac{1}{r^{2}} \frac{\partial^{2} \phi}{\partial \theta^{2}}+\frac{\partial^{2} \phi}{\partial z^{2}}=0 .
$$

Because the fluid is inviscid, the boundary conditions at the side walls and the bottom are respectively given by

$$
\left.\frac{\partial \phi}{\partial r}\right|_{r=R}=0 \quad \text { and }\left.\quad \frac{\partial \phi}{\partial z}\right|_{z=-h}=0 .
$$

Because the dynamic, free-surface boundary condition is the requirement that the pressure at the free surface be the constant atmospheric pressure, we apply the unsteady, irrotational Bernoulli equation at the free surface:

$$
\frac{\partial \phi}{\partial t}+\frac{1}{2}|\nabla \phi|^{2}+\frac{p}{\rho}+\Gamma=f(t)
$$

where

$$
\Gamma=-\frac{a r}{2}\left[(\omega+\xi)^{2} e^{i((\omega+\xi) t-\theta\}}+(\omega-\xi)^{2} e^{i\{(\omega-\xi) t+\theta\}}\right]+g z,
$$

and $f(t)$ is an arbitrary function of time. Here $p$ is the pressure, $\rho$ is the liquid density, and $g$ is the gravitational acceleration. $\Gamma$ is the potential of the external force that is represented by inertial and gravitational forces. Because the fluid velocity is assumed to be small and the rotating velocity of the cylindrical vessel is constant, Coriolis force and the force caused by the rotational motion are considered to be infinitesimally small. Then Eq. (3) becomes

$$
\left(\frac{\partial^{2} \phi}{\partial t^{2}}+g \frac{\partial \phi}{\partial z}\right)_{z=0}=\frac{i a r}{2}\left[(\omega+\xi)^{3} e^{i\{(\omega+\xi) t-\theta\}}+(\omega-\xi)^{3} e^{i\{(\omega-\xi) t+\theta\}}\right] .
$$

Solving Eq. (1) using the given boundary conditions, we obtain

$$
\begin{aligned}
\phi=\sum_{m=1}^{\infty} \sum_{n=1}^{\infty}[ & \cosh \left\{k_{m n}(z+h)\right\}\left\{F_{m n} J_{m}\left(k_{m n} r\right) \cos \left(m \theta+\delta_{m}\right) \cos \left(\omega_{m n} t+\epsilon_{m n}\right)\right. \\
& -\frac{a r}{2 \cosh \left(k_{m n} h\right)}\left[\frac{(\omega+\xi)^{3}}{\omega_{m n}^{2}-(\omega+\xi)^{2}} \sin \{(\omega+\xi) t-\theta\}\right. \\
& \left.\left.\left.+\frac{(\omega-\xi)^{3}}{\omega_{m n}^{2}-(\omega-\xi)^{2}} \sin \{(\omega-\xi) t+\theta\}\right]\right\}\right],
\end{aligned}
$$


where $m$ and $n$ are integers $(m=1,2,3, \cdots, n=1,2,3, \cdots)$ that represent the $(m, n)$ oscillation mode. Further, $J_{m}$ is the Bessel function of the first kind of order $m$, and $F_{m n}, \delta_{m n}$, and $\epsilon_{m n}$ are arbitrary constants. The quantities $k_{m n}$ and $\omega_{m n}$ are constants that satisfy the following equations:

$$
\left.\frac{d J_{m}\left(k_{m n} r\right)}{d r}\right|_{r=R}=0, \quad \text { and } \quad \omega_{m n}=\sqrt{g k_{m n} \tanh \left(k_{m n} h\right)}
$$

From Eqs. (3), (4) and (6), we obtain the pressure fluctuation $\Delta p$ as

$$
\begin{aligned}
\Delta p & =\underbrace{2 \pi^{2} \rho \operatorname{arr}(f+\Omega)^{2}\left\{\sum_{m=1}^{\infty} \sum_{n=1}^{\infty} \frac{\cosh \left\{k_{m n}(z+h)\right\}(f+\Omega)^{2}}{\cosh \left(k_{m n} h\right)\left\{f_{m n}^{2}-(f+\Omega)^{2}\right\}}+1\right\} \cos \{\theta-2 \pi(f+\Omega) t\}}_{\Delta p_{r}} \\
& +\underbrace{2 \pi^{2} \rho \operatorname{ar}(f-\Omega)^{2}\left\{\sum_{m=1}^{\infty} \sum_{n=1}^{\infty} \frac{\cosh \left\{k_{m n}(z+h)\right\}(f-\Omega)^{2}}{\cosh \left(k_{m n} h\right)\left\{f_{m n}^{2}-(f-\Omega)^{2}\right\}}+1\right\} \cos \{\theta+2 \pi(f-\Omega) t\}}_{\Delta p_{f}} .
\end{aligned}
$$

Here, the angular frequencies are replaced by the normal-mode frequencies for the convenience of the experiment:

$$
\omega=2 \pi f, \quad \xi=2 \pi \Omega, \quad \text { and } \quad \omega_{m n}=2 \pi f_{m n} .
$$

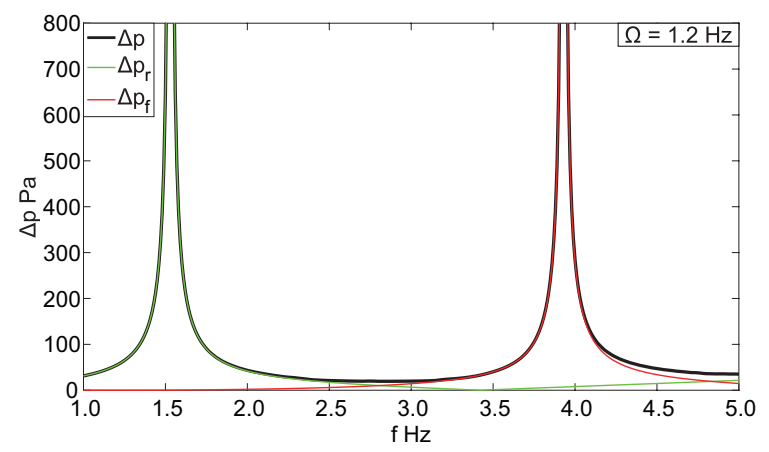

Figure 3. Theoretical frequency response of the pressure fluctuation

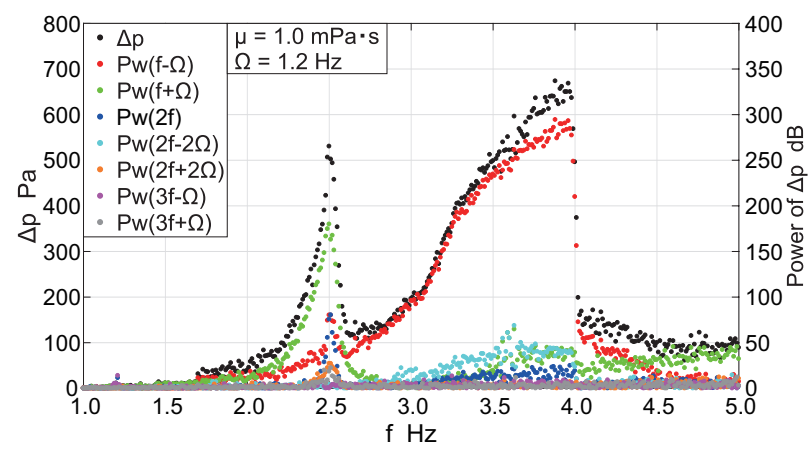

Figure 4. Frequency response of the pressure fluctuation for viscosity $\mu=1.0 \mathrm{mPa} \cdot \mathrm{s}$ and $\Omega=1.2 \mathrm{~Hz}$. 
The quantity $\Delta p$ has many frequency components, as indicated in Eq. (8). The dominant frequencies correspond to the mode with $m=1$ and $n=1$. Figure 3 shows the frequency response $\Delta p$ predicted for the location of the pressure sensor $(z=-17 \mathrm{~mm})$ for a rotation frequency $\Omega=1.2 \mathrm{~Hz}$. Here $m=1$ and $n=1$. There are two resonant frequencies, which correspond to the terms $\Delta p_{r}$ and $\Delta p_{f}$ given in Eq. (8).

\section{Results and discussions}

Figures 4 shows the experimental results for the frequency response of the pressure fluctuation $\Delta p$ for a viscosity $\mu=1.0 \mathrm{mPa} \cdot \mathrm{s}$; the test liquid is only water in this case. The quantity $\Delta p$ has two resonant frequencies, as given by the theoretical results shown in Fig. 3 . When the forcing frequency $f$ increases to the first resonant frequency, swirling of the free surface occurs, but with a rotational direction opposite to that of the cylindrical vessel. When the forcing frequency passes the first resonant frequency, the swirling disappears, and the oscillation of the free surface becomes like a standing wave. As the forcing frequency increases, approaching the second resonant frequency, swirling of the free surface appears again, but in this case the direction of rotation is the same as that of the cylindrical vessel. The swirling

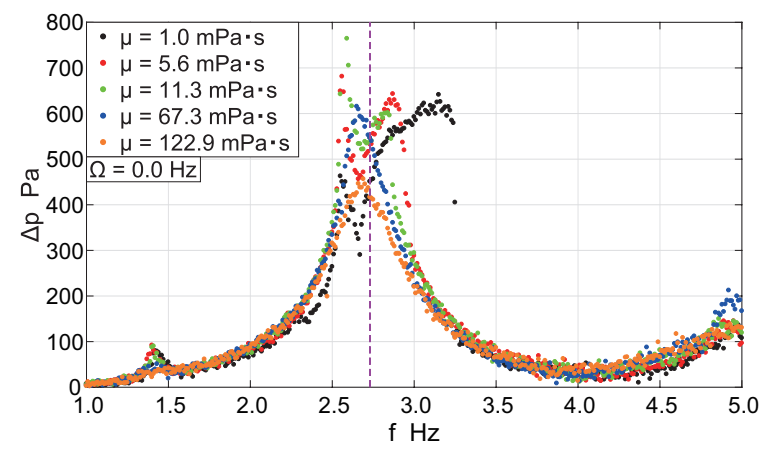

Figure 5. Frequency response of the pressure fluctuation for $\Omega=0.0 \mathrm{~Hz}$ with liquids of different viscosities

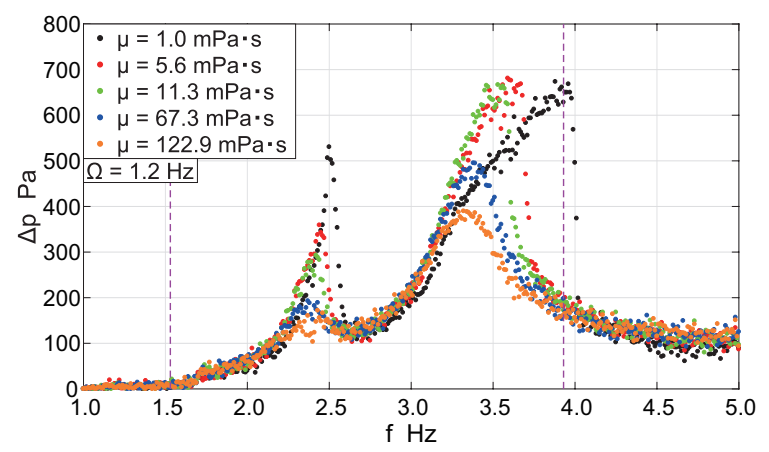

Figure 6. Frequency response of the pressure fluctuation for $\Omega=1.2 \mathrm{~Hz}$ with liquids of different viscosities 
abruptly disappears when the forcing frequency passes the second resonant frequency. From the FFT analysis, we found that $\Delta p$ has several frequency components. Some typical points in the power spectra of $\Delta p$ are denoted by $P_{w}$ in Fig. 4 . From the theoretical analysis, we expect the dominant frequencies to be $f+\Omega$ and $f-\Omega$, which correspond to the terms $\Delta p_{r}$ and $\Delta p_{f}$ in Fig. 3. The second resonant frequency in Fig. 4 is roughly the same as the theoretical result, $f-\Omega$, but the first resonant frequency, is appreciably different from the theoretical result, $f+\Omega$.

Figure 5 shows the frequency response of the pressure fluctuations for liquids with different viscosities when the cylindrical vessel is subjected only to lateral excitation but is not rotated: $\Omega=0$; the dashed line indicates the theoretical resonance frequency. For these experiments, the pressure sensor was located at $\theta=0$ (see Fig. 2). For low-viscosity liquids, the direction of rotation of the swirl changes irregularly around $f=2.55 \mathrm{~Hz}$, and the resonant frequency exceeds the theoretical resonant frequency. On the other hand, irregular swirling does not occur for high-viscosity liquids, and the resonant frequency is close to the theoretical resonant frequency.

Figure 6 shows the frequency response of the pressure fluctuations for liquids with different viscosities at the rotation frequency $\Omega=1.2 \mathrm{~Hz}$. When the cylindrical vessel is rotating, two resonance frequencies appear. The value of the first resonant frequency differs substantially from the theoretical result, irrespective of the viscosity. This indicates that the rotation of the cylindrical vessel has a much greater effect on the frequency response than does the viscosity. Conversely, the second resonant frequency is almost the same as the result for the low-viscosity liquids, but the resonant frequencies vary with viscosity for the high-viscosity liquids. This suggests that the resistance force between the cylindrical vessel and the liquid changes with the liquid viscosity.

\section{Concluding remarks}

We have investigated the frequency response of the dynamic pressure fluctuations for mixtures of water and glycerin in a rotating, laterally oscillating, cylindrical vessel. Sloshing of the liquid can be suppressed, and unstable swirling motions can be eliminated, by adding rotational motion to the lateral oscillation of the vessel. From a linearized analysis, we found two predominant frequencies. Two dominant frequencies also occurred in the experiments, together with other frequency components found by the FFT analysis. These frequency components are combinations of the lateral-oscillation and rotational frequencies. However, we have not been able to clarify the detailed physical meaning of these frequencies in the present work.

\section{References}

[1] R. E. Hutton, NASA Tech. Note D-1870, 1 (1963)

[2] M. L. Rocca, G. Sciortino, M. A. Boniforti, Fluid Dyn. Res. 27, 23 (2000)

[3] H. F. Bauer, W. Eidel, Forschung im Ingenieurwesen 67, 93 (2002)

[4] H. Takahara, K. Kimura, J. Sound Vib. 331, 3199 (2012)

[5] R. A. Ibrahim, V. N. Pilipchuk, T. Ikeda, Appl. Mech. Rev. 54, 133 (2001)

[6] R. A. Ibrahim, Liquid sloshing dynamics (Cambridge University Press, New York, 2005)

[7] M. Ohaba, T. Sawada, S. Sudo, T. Tanahashi, Proc. JSME Fluids Eng. Conf., 203 (1995)

[8] Y. Saito, T. Sawada, Unvrsl. J. Mech. Eng. 5, 97 (2017). 